\title{
Resistance of the stable-towards more precise prediction of response to immune checkpoint blockade in microsatellite-unstable cancer patients
}

\author{
Matthias Kloor ${ }^{1,2}$ \\ ${ }^{1}$ Department of Applied Tumor Biology, Institute of Pathology, University Hospital Heidelberg, Heidelberg, Germany; ${ }^{2}$ Clinical Cooperation Unit \\ Applied Tumor Biology, DKFZ (German Cancer Research Center) Heidelberg, Heidelberg, Germany \\ Correspondence to: PD Dr. med. Matthias Kloor. Department of Applied Tumor Biology, Institute of Pathology, University Hospital Heidelberg, Im \\ Neuenheimer Feld 224, 69120 Heidelberg, Germany. Email: matthias.kloor@med.uni-heidelberg.de. \\ Provenance: This is an invited article commissioned by the Academic Editor Dr. Jingping Wang (Department of Pathology, The First Affiliated \\ Hospital of Bengbu Medical College, Bengbu Medical College, Bengbu, China). \\ Comment on: Cohen R, Hain E, Buhard O, et al. Association of Primary Resistance to Immune Checkpoint Inhibitors in Metastatic Colorectal Cancer \\ With Misdiagnosis of Microsatellite Instability or Mismatch Repair Deficiency Status. JAMA Oncol 2019;5:551-5.
}

Submitted Sep 20, 2019. Accepted for publication Sep 30, 2019.

doi: $10.21037 /$ atm.2019.10.29

View this article at: http://dx.doi.org/10.21037/atm.2019.10.29

The introduction of immune checkpoint blockade into the clinical practice has marked a milestone for treating patients with advanced microsatellite-unstable (MSI) cancer $(1,2)$. MSI tumors are evolving with a deficiency of the DNA mismatch repair (MMR) system and therefore accumulate numerous somatic mutations during every cell division, resulting in a very high mutational load in the manifest tumors $(3,4)$. The majority of these mutations seem to affect short repetitive sequence stretches (microsatellites) as insertion or, mostly, deletion of single nucleotides. When microsatellites located in gene-encoding regions are affected by indel mutations, translational frame shifts occur, a process that can give rise to the generation of mutational neoantigens $(5,6)$.

The high frameshift peptide neoantigen load apparently allows efficient recognition of MSI tumor cells by the immune system (7). T cells specific for MSI-related neoantigens have been detected in the peripheral blood drawn from MSI cancer patients (8), and the cytotoxic potential of such $\mathrm{T}$ cells has been demonstrated (9). Immune surveillance is most likely responsible for the rather favorable prognosis and lower stage of MSI compared to microsatellite-stable cancers (5). In spite of immune surveillance typically observed in MSI tumors $(10,11)$, a subset of MSI cancers acquire a phenotype that enables formation of distant metastasis. In patients with metastasized MSI cancer, immune checkpoint blockade can help to reactivate an exhausted immune system and unleash a more forceful anti-tumor immune attack, often resulting in reduction of the tumor burden and, in a substantial proportion of patients, even in complete responses, i.e., elimination of any detectable tumor lesions (2).

Although immune checkpoint blockade shows remarkable success in the treatment of patients with metastasized MSI cancer, therapy success is not universal, as some patients develop progressive disease under therapy and do not have any benefit from treatment. Importantly, accurate and reliable prediction of therapy response for individual MSI cancer patients is still not possible.

\section{Possible mechanisms of resistance}

Several factors may contribute to resistance towards immune checkpoint blockade. Resistance to checkpoint blockade in patients with malignant melanoma has been related to a breakdown of the cellular antigen presentation machinery in tumor cells (12), mainly mediated by Beta-2microglobulin (B2M) mutations or loss of heterozygosity. The human $B 2 M$ gene codes for a small protein of 119 amino acids in length, which is essential for the correct assembly of HLA class I antigen complexes on the cell surface (13). If $B 2 M$ is mutant in tumor cells, most likely as the result of 
immunoselection, tumor cells cannot present intracellular antigens via HLA class I antigens to T cells with cytotoxic potential (14). Consequently, immune checkpoint blockade may fail, because treatment-activated $\mathrm{T}$ cells will not recognize the intended target cells (15). MSI colorectal cancers harbor $B 2 M$ mutations in about $30 \%$ of the lesions, albeit at a significantly lower frequency in MSI cancers with distant metastases $(16,17)$. Recently, favorable outcomes of $B 2 M$-mutant cancer patients receiving immune checkpoint blockade have been reported (18); the mechanisms underlying this observation need further investigation.

In addition to $B 2 M$ mutations, breakdown of the antigen presentation machinery potentially abrogating the success of immune checkpoint blockade can be mediated by other mutations affecting essential components of the antigen processing and presentation machinery, and many of them have been reported in MSI cancer cells (19).

In tumors with the antigen presentation machinery intact, lack of activated immune cells or an insufficient amount of neoantigens may confer resistance. This is most likely the reason for the inefficiency of immune checkpoint blockade in MSS colorectal cancers and other tumor types with a low neoantigen load (1). A recent study also suggests that among MSI tumors those with a relatively limited number of neoantigens may have a lower likelihood of clinical response (20).

The paper published by Cohen et al. (21) now provides a different and notable explanation for non-responsiveness towards immune checkpoint blockade: Among five patients with primary resistance towards immune checkpoint blockade, re-evaluation revealed misdiagnosis of MSI in three tumors that were actually microsatellite-stable and MMR-proficient. Although the number of tumors analyzed in this study was limited, the results clearly demonstrate the need for quality-controlled test settings to ensure proper classification of tumors as MSI.

\section{Significance of MSI testing}

Reliability and validity of MSI testing have been a matter of debate virtually since the discovery of the MSI phenotype related to MMR deficiency in 1993 (22,23). However, the consequences of false MSI testing results have reached an entirely different level of immediacy with the clinical introduction of immune checkpoint inhibitors for treating metastasized MSI tumors irrespective of the anatomic location and origin of the tumor.

Now, false classification of a tumor as MSI can expose patients to a therapy with potentially significant side effects (24) while at the same time not providing a relevant benefit. The consequences of false classification of an MSI tumor as MSS are no less severe: a patient may not be offered a potentially life-saving treatment, robbing her or him of the improved chance of survival otherwise provided by the newly developed immune checkpoint inhibitors.

The study by Cohen et al. indicates that error rates of MSI testing potentially tolerable in a purely scientific setting have to be reconsidered for the clinical setting. Moreover, errors resulting from false-positive classification of a tumor as MSI even more dramatically reduces the positive predictive value in the scenario of metastasized MSI cancer, because the prevalence of the MSI phenotype is lower in metastasized than in unselected cancers.

\section{Pitfalls of MSI testing}

In principle, two approaches are available to evaluate MSI. First, the expression of MMR proteins can be assessed by immunohistochemistry; second, the functionality of the MMR system can be evaluated by PCR-based evaluation of microsatellite lengths, which are expected to deviate from their normal length if the MMR system is inactive. While immunohistochemistry can indicate the presence or absence of MMR proteins in tumor cell nuclei, PCR-based MSI testing more directly examines whether MMR functionality was present or absent during tumor evolution. Both methods have advantages and disadvantages, some of which are exemplarily illustrated in the publication by Cohen et al.

Due to high inter-laboratory variation regarding methods used and interpretation of the results, consensus conferences have suggested standard procedures to be followed for proper classification of MSI, mainly in order to identify patients affected by inherited pathogenic MMR gene variants (Lynch syndrome) $(25,26)$.

Studying the scientific literature clearly shows that non-adherence to these recommendations leads to wrong estimations of the MSI prevalence in different tumor types, sometimes with unrealistically high percentages. To avoid pitfalls related to PCR-based MSI testing, mononucleotide markers should be used, which are clearly more sensitive and specific indicators of MMR deficiency than di- or tetranucleotide markers $(27,28)$. Moreover, mononucleotide markers, including the "classical" MSI markers BAT25 or BAT26 or subsequently published markers $(29,30)$, are quasimonomorphic in Caucasian and Asian populations. This advantage allows their use also when no normal tissue is 
available as a reference. Interestingly, in the study by Cohen et al. (21), false positive classification of a tumor as MSI occurred in a patient of African origin who harbored variant alleles of three of the tested markers. This observation clearly suggests that PCR-based MSI testing should include a normal tissue reference whenever discrepant results are observed between PCR and immunohistochemistry, or when only part of the analyzed PCR markers display variant alleles potentially indicating MSI.

The risk of false classification of a tumor as MSS is particularly high if immunohistochemistry staining of the MMR proteins MLH1 and MSH2 is used as a single method for MSI classification (31). When tumors are stained for all four MMR proteins including MSH6 and PMS2, as should inevitably be done, the likelihood of missing MSI can be reduced; however, equivocal situations can still occur, related either to (mainly extracolonic) tumors with a low proliferation rate, tissue fixation artifacts or other technical limitations that interfere with the interpretability of immunohistochemistry $(32,33)$. Interpretation of MMR protein immunohistochemistry requires experience and training, including the necessity of correctly identifying nuclear staining status of tumor cells, correct cross-evaluation of MMR proteins that are organized in heterodimers, and only calling MMR loss in a tumor if normal reference cells show positive staining.

At present, using both, immunohistochemistry staining of MMR proteins and PCR-based evaluation of MSI using mononucleotide markers as recommended by Cohen et al. (21) represents the most solid and robust approach to ensure proper MSI diagnostics in the clinical setting.

\section{Perspectives}

For sure, it is difficult to derive definitive recommendations on concrete standard procedures for MSI evaluation from the study. Although it may seem natural to postulate more studies comparatively applying different test methods in the clinical routine, designing a reasonable scenario for their realization is difficult. What about new tests for determining MSI, either using more sensitive markers or applying next generation-based testing methods? What are the reliability and the validity of these methods? Even if hypothetically a higher sensitivity may be achieved, would this be desirable? Would patients who are reclassified as MSI with novel, potentially more sensitive tools still respond to immune checkpoint blockade, or is a certain level of instability, possibly corresponding to the general mutational burden, required for recognition of tumor cells by the immune system? These questions require careful assessment (28), as the medical need for accurate classification is evident.

The study by Cohen et al., in line with other publications $(2,34)$, also indicates that resistance to immune checkpoint blockade can also occur in "true" MSI, MMR-deficient cancer patients. This highlights the need for further translational research on factors responsible for therapy resistance or responsiveness. All available tools from basic immunology, in vitro and in vivo should be used to provide reasonable hypotheses and evaluate them in order to guide the focus of research accompanying future clinical studies. Most importantly, the identification of reliable biomarkers for prediction of response to immune checkpoint blockade in MSI cancer patients, but also beyond, clearly requires multicenter efforts, ensuring that all available information is gathered and not lost due to differing protocols between separate studies of different designs.

In summary, the instructive publication by Cohen et al. highlights the need for quality control and appropriate diagnostics procedures for MSI classification of tumors. Highest possible quality standards and expertise are required to ensure optimal guidance for patients potentially eligible for immune checkpoint blockade. In addition, comprehensive documentation of clinical and molecular data is warranted and required to obtain more profound information about potential predictors of response or resistance, across centers and across trials. Information about molecular phenotypes of the tumor, its antigen presentation capacity as well as the local and systemic immune environment should be recorded for all patients receiving immune checkpoint blockade whenever possible.

\section{Acknowledgments}

None.

\section{Footnote}

Conflicts of Interest: The author has no conflicts of interest to declare.

Ethical Statement: The author is accountable for all aspects of the work in ensuring that questions related to the accuracy or integrity of any part of the work are appropriately investigated and resolved. 


\section{References}

1. Le DT, Uram JN, Wang H, et al. PD-1 Blockade in Tumors with Mismatch-Repair Deficiency. N Engl J Med 2015;372:2509-20.

2. Le DT, Durham JN, Smith KN, et al. Mismatch repair deficiency predicts response of solid tumors to PD-1 blockade. Science 2017;357:409-13.

3. Salem ME, Puccini A, Grothey A, et al. Landscape of Tumor Mutation Load, Mismatch Repair Deficiency, and PD-L1 Expression in a Large Patient Cohort of Gastrointestinal Cancers. Mol Cancer Res 2018;16:805-12.

4. Network CGA. Comprehensive molecular characterization of human colon and rectal cancer. Nature 2012;487:330-7.

5. Kloor M, von Knebel Doeberitz M. The Immune Biology of Microsatellite-Unstable Cancer. Trends Cancer 2016;2:121-33.

6. Saeterdal I, Bjorheim J, Lislerud K, et al. Frameshiftmutation-derived peptides as tumor-specific antigens in inherited and spontaneous colorectal cancer. Proc Natl Acad Sci U S A 2001;98:13255-60.

7. Maby P, Tougeron D, Hamieh M, et al. Correlation between Density of CD8+ T-cell Infiltrate in Microsatellite Unstable Colorectal Cancers and Frameshift Mutations: A Rationale for Personalized Immunotherapy. Cancer Res 2015;75:3446-55.

8. Schwitalle Y, Kloor M, Eiermann S, et al. Immune response against frameshift-induced neopeptides in HNPCC patients and healthy HNPCC mutation carriers. Gastroenterology 2008;134:988-97.

9. Linnebacher M, Gebert J, Rudy W, et al. Frameshift peptide-derived T-cell epitopes: a source of novel tumorspecific antigens. Int J Cancer 2001;93:6-11.

10. Buckowitz A, Knaebel HP, Benner A, et al. Microsatellite instability in colorectal cancer is associated with local lymphocyte infiltration and low frequency of distant metastases. Br J Cancer 2005;92:1746-53.

11. Dolcetti R, Viel A, Doglioni C, et al. High prevalence of activated intraepithelial cytotoxic $\mathrm{T}$ lymphocytes and increased neoplastic cell apoptosis in colorectal carcinomas with microsatellite instability. Am J Pathol 1999; 154:1805-13.

12. Sade-Feldman M, Jiao YJ, Chen JH, et al. Resistance to checkpoint blockade therapy through inactivation of antigen presentation. Nat Commun 2017;8:1136.

13. Ploegh HL, Orr HT, Strominger JL. Major histocompatibility antigens: the human (HLA-A, -B, -C) and murine (H-2K, H-2D) class I molecules. Cell
1981;24:287-99.

14. Sucker A, Zhao F, Real B, et al. Genetic evolution of T-cell resistance in the course of melanoma progression. Clin Cancer Res 2014;20:6593-604.

15. Gettinger S, Choi J, Hastings K, et al. Impaired HLA Class I Antigen Processing and Presentation as a Mechanism of Acquired Resistance to Immune Checkpoint Inhibitors in Lung Cancer. Cancer Discov 2017;7:1420-35.

16. Kloor M, Michel S, Buckowitz B, et al. Beta2microglobulin mutations in microsatellite unstable colorectal tumors. Int J Cancer 2007;121:454-8.

17. Kloor M, Michel S, von Knebel Doeberitz M. Immune evasion of microsatellite unstable colorectal cancers. Int J Cancer 2010;127:1001-10.

18. Middha S, Yaeger R, Shia J, et al. Majority of B2M-Mutant and -Deficient Colorectal Carcinomas Achieve Clinical Benefit From Immune Checkpoint Inhibitor Therapy and Are Microsatellite Instability-High. JCO Precis Oncol 2019. doi: 10.1200/PO.18.00321.

19. Ozcan M, Janikovits J, von Knebel Doeberitz M, et al. Complex pattern of immune evasion in MSI colorectal cancer. Oncoimmunology 2018;7:e1445453.

20. Mandal R, Samstein RM, Lee KW, et al. Genetic diversity of tumors with mismatch repair deficiency influences antiPD-1 immunotherapy response. Science 2019;364:485-91.

21. Cohen R, Hain E, Buhard O, et al. Association of Primary Resistance to Immune Checkpoint Inhibitors in Metastatic Colorectal Cancer With Misdiagnosis of Microsatellite Instability or Mismatch Repair Deficiency Status. JAMA Oncol 2019;5:551-5.

22. Ionov Y, Peinado MA, Malkhosyan S, et al. Ubiquitous somatic mutations in simple repeated sequences reveal a new mechanism for colonic carcinogenesis. Nature 1993;363:558-61.

23. Thibodeau SN, Bren G, Schaid D. Microsatellite instability in cancer of the proximal colon. Science 1993;260:816-9.

24. Tauber M, Cohen R, Laly P, et al. Severe necrotizing myositis associated with long term anti-neoplastic efficacy following nivolumab plus ipilimumab combination therapy. Clin Rheumatol 2019;38:601-2.

25. Umar A, Boland CR, Terdiman JP, et al. Revised Bethesda Guidelines for hereditary nonpolyposis colorectal cancer (Lynch syndrome) and microsatellite instability. J Natl Cancer Inst 2004;96:261-8.

26. Boland CR, Thibodeau SN, Hamilton SR, et al. A National Cancer Institute Workshop on Microsatellite 
Instability for cancer detection and familial predisposition: development of international criteria for the determination of microsatellite instability in colorectal cancer. Cancer Res 1998;58:5248-57.

27. Bacher JW, Abdel Megid WM, Kent-First MG, et al. Use of mononucleotide repeat markers for detection of microsatellite instability in mouse tumors. Mol Carcinog 2005;44:285-92.

28. Luchini C, Bibeau F, Ligtenberg MJL, et al. ESMO recommendations on microsatellite instability testing for immunotherapy in cancer, and its relationship with PD-1/ PD-L1 expression and tumour mutational burden: a systematic review-based approach. Ann Oncol 2019. [Epub ahead of print].

29. Findeisen P, Kloor M, Merx S, et al. T25 repeat in the $3^{\prime}$ untranslated region of the CASP2 gene: a sensitive and specific marker for microsatellite instability in colorectal cancer. Cancer Res 2005;65:8072-8.

30. Suraweera N, Duval A, Reperant M, et al. Evaluation of tumor microsatellite instability using five

Cite this article as: Kloor M. Resistance of the stable-towards more precise prediction of response to immune checkpoint blockade in microsatellite-unstable cancer patients. Ann Transl Med 2019;7(21):603. doi: 10.21037/atm.2019.10.29 quasimonomorphic mononucleotide repeats and pentaplex PCR. Gastroenterology 2002;123:1804-11.

31. Lindor NM, Burgart LJ, Leontovich O, et al. Immunohistochemistry versus microsatellite instability testing in phenotyping colorectal tumors. J Clin Oncol 2002;20:1043-8.

32. Svrcek M, Lascols O, Cohen R, et al. MSI/MMRdeficient tumor diagnosis: Which standard for screening and for diagnosis? Diagnostic modalities for the colon and other sites: Differences between tumors. Bull Cancer 2019;106:119-28.

33. Shia J. Immunohistochemistry versus microsatellite instability testing for screening colorectal cancer patients at risk for hereditary nonpolyposis colorectal cancer syndrome. Part I. The utility of immunohistochemistry. J Mol Diagn 2008;10:293-300.

34. Overman MJ, Lonardi S, Wong KYM, et al. Durable Clinical Benefit With Nivolumab Plus Ipilimumab in DNA Mismatch Repair-Deficient/Microsatellite Instability-High Metastatic Colorectal Cancer. J Clin Oncol 2018;36:773-9. 